\title{
@ Neuroanatomical abnormalities before and after onset of psychosis: a cross-sectional and longitudinal MRI comparison
}

Christos Pantelis, Dennis Velakoulis, Patrick D McGorry, Stephen J Wood, John Suckling, Lisa J Phillips, Alison R Yung,

Edward T Bullmore, Warrick Brewer, Bridget Soulsby, Patricia Desmond, Philip K McGuire

\begin{abstract}
Summary
Background Psychotic disorders, such as schizophrenia, are associated with neuroanatomical abnormalities, but whether these predate the onset of symptoms or develop progressively over the course of illness is unclear. We investigated this issue with MRI to study people with prodromal symptoms who are at ultra high-risk for the development of psychosis.
\end{abstract}

Methods We did two comparisons, cross-sectional and longitudinal. For the cross-sectional comparison, 75 people with prodromal signs of psychosis were scanned with MRI. After at least 12 months of follow-up, 23 (31\%) had developed psychosis and $52(69 \%)$ had not. Baseline MRI data from these two subgroups were compared. For the longitudinal comparison, 21 of the ultra high-risk individuals were scanned again with MRI after at least 12 months. Ten of these had developed psychosis and 11 had not. MRI data from baseline and follow-up were compared within each group of people.

Findings In the cross-sectional comparison, compared with people who did not develop psychosis, those who did develop the disorder had less grey matter in the right medial temporal, lateral temporal, and inferior frontal cortex, and in the cingulate cortex bilaterally. In the longitudinal comparison, when re-scanned, individuals who had developed psychosis showed a reduction in grey matter in the left parahippocampal, fusiform, orbitofrontal and cerebellar cortices, and the cingulate gyri. In those who had

Cognitive Neuropsychiatry Research and Academic Unit, and Melbourne Neuropsychiatry Centre, Mental Health Programme, Department of Psychiatry, University of Melbourne and Sunshine Hospital, Melbourne, Victoria, Australia (C Pantelis FRANZCP, D Velakoulis FRANZCP, S J Wood PhD, W Brewer PhD, B Soulsby BSc); Applied Schizophrenia Division, Mental Health Research Institute of Victoria, Melbourne (C Pantelis, D Velakoulis, S J Wood, W Brewer, B Soulsby); Early Psychosis Prevention and Intervention Centre, Personal Assessment and Crisis Evaluation Clinic, and Department of Psychiatry, University of Melbourne, Melbourne (Prof P D McGorry FRAnZCP, L J Phillips MPsych (Clin), A R Yung FRANZCP); Department of Radiology, University of Melbourne and Royal Melbourne Hospital, Melbourne (P Desmond FRACR); Clinical Age Research Unit, Guy's, King's, and St Thomas' Medical School, London, UK (J Suckling PhD); Brain Mapping Unit, Department of Psychiatry, University of Cambridge, Cambridge (Prof E T Bullmore MRCPsych); Section of Neuroimaging, Institute of Psychiatry, London (Prof P K McGuire MRCPsych); and Outreach and Support in South London, South London and Maudsley NHS Trust, London (Prof P K McGuire)

Correspondence to: Dr Christos Pantelis, Cognitive Neuropsychiatry Research and Academic Unit, Sunshine Hospital, PO Box 294, St Albans, Victoria 3021, Australia

(e-mail: cpant@unimelb.edu.au) not become psychotic, longitudinal changes were restricted to the cerebellum.

Interpretation Some of the grey-matter abnormalities associated with psychotic disorders predate the onset of frank symptoms, whereas others appear in association with their first expression.

Published online December 10, 2002

http://image.thelancet.com/extras/01art9092web.pdf See Commentary

\section{Introduction}

Psychotic disorders involve striking disturbances of thought, perception, affect, and behaviour. The major psychotic disorders, especially schizophrenia and bipolar disorder, are relatively undifferentiated during the early phase of illness, so that to distinguish them at this stage can be difficult. ${ }^{1}$ However, studies done early in the course of psychosis are essential for an understanding of the neurobiology and treatment of these disorders.

Although increasing consensus exists that the structure of the brain is abnormal in major psychotic disorders, whether these abnormalities predate onset of illness and are relatively fixed over its course, or whether they are progressive, remains controversial. ${ }^{2}$ Results of neuroimaging studies suggest that abnormalities that are qualitatively similar to those evident in established schizophrenia and bipolar disorder are apparent in patients presenting with their first episode of psychosis ${ }^{3-6}$ and in individuals without psychosis with a strong familial risk of schizophrenia. ${ }^{7}$ However, results of recent longitudinal neuroimaging studies in patients with schizophrenia have shown structural changes within the same patients over time, especially in the early stages of illness. ${ }^{8}$ These two sets of findings raise the possibility that neuroanatomical abnormalities seen in patients with psychotic disorders reflect a combination of pre-existing vulnerability and changes associated with the first expression of psychotic symptoms.

We aimed to investigate this model of psychosis with MRI, to assess people at ultra high-risk of development of psychosis before and after onset of their first episode of psychosis. On the basis of our own and other work, we predicted that ultra high-risk people would show volumetric abnormalities before they became psychotic, and that these would be qualitatively similar to those seen in patients with established schizophrenia and bipolar disorder..$^{3-6}$ A second prediction was that there would also be progressive loss of grey matter in the medial temporal cortex associated with development of psychosis in ultra high-risk indviduals. This hypothesis was based on findings of smaller medial temporal volumes in patients with psychosis than in ultra high-risk individuals. ${ }^{7,9}$ 


\section{Participants and methods}

Participants

We recruited people at ultra high-risk of development of psychosis from the personal assessment and crisis evaluation (PACE) clinic in Melbourne, Australia, which manages young people at risk of this disorder. ${ }^{10}$ We used a close-in strategy to identify individuals at ultra high-risk of psychosis with a combination of trait and state factors (panel). ${ }^{10}$ This strategy identified people whose theoretical risk of becoming psychotic was $41 \%$ within a 12-month period. ${ }^{10} \mathrm{We}$ enrolled all individuals at this clinic who gave written informed consent to be scanned and who met our inclusion criteria, including adequate clinical follow-up to confirm diagnostic outcome. We excluded individuals if they had a history of head injury, seizures, cerebrovascular disease, other neurological disease, impaired thyroid function, or steroid abuse. At enrolment, we assessed all participants with the brief psychiatric rating scale (BPRS), scale for the assessment of negative symptoms (SANS), and national adult reading test (NART). The study was approved by the local research and ethics committee.

\section{Procedures}

In the first, cross-sectional comparison, we scanned individuals at baseline and then followed them up clinically for 24 months. We compared MRI data from the subgroup of individuals who subsequently developed psychosis within the follow-up period with data from those who did not develop this disorder. We did the crosssectional comparison to show whether volumetric MRI differences between the two groups were evident before onset of psychosis.
In people who developed psychosis, we assessed the type of psychotic disorder with the structured clinical interview for the diagnostic and statistical manual of mental disorders, 4 th edition (SCID).

In the second, longitudinal comparison, we rescanned a subset of individuals from the cross-sectional comparison with the same MRI protocol (see below). This subset of people included all those in whom follow-up scanning was possible. We compared MRI scans done at baseline with those done at follow-up. This longitudinal within-subject comparison showed whether transition from the ultra highrisk to the psychotic state was associated with progressive changes in grey-matter volume.

We acquired high-resolution 3-mm thick, contiguous, interleaved, two-dimensional, dual-echo fast spin-echo images parallel to the AC-PC (anterior and posterior commissure) line, covering the entire brain. Proton density (PD)-weighted and T2-weighted images were acquired almost simultaneously (echo time 1, $20 \mathrm{~ms}$; echo time 2 , $85 \mathrm{~ms}$; repetition time, $4000 \mathrm{~ms}$; eight-echo train length). We set the matrix size and field of view at $256 \times 192$, and $22 \mathrm{~cm}$, respectively, and every voxel was represented with a 16 -bit integer value.

The methods we used for segmentation and registration of each image dataset have been described in detail elsewhere. ${ }^{11,12}$ Briefly, voxels representing extracerebral tissue were automatically identified and set to zero with a linear scale space set of features obtained from derivatives of the Gaussian kernel. ${ }^{11}$ Manual editing of the segmented images was only necessary to remove brainstem from the cerebral hemispheres and diencephalon. We then estimated the probability of each intracerebral voxel belonging to each of four possible tissue classes (grey

\section{Intake and exit criteria}

\section{Intake criteria}

Group 1: attenuated psychotic symptoms

- Presence of at least one of the following symptoms: ideas of reference, magical thinking, perceptual disturbance, paranoid ideation, odd thinking and speech (2-3 on unusual thought content scale; $1-2$ on hallucinations scale; $2-3$ on suspiciousness scale; or 1-3 on conceptual disorganisation scale of BPRS)

- Held with a reasonable degree of conviction, as defined by a score of 2 on the CASH rating scale for delusions

- Frequency of symptoms: several times per week

- Change in mental state present for at least 1 week and not longer than 5 years

Group 2: brief limited intermittent psychotic symptoms

- Transient psychotic symptoms: ideas of reference, magical thinking, perceptual disturbance, paranoid ideation, odd thinking and speech (4+ on unusual thought content scale; $3+$ on hallucinations scale; 4+ on suspiciousness scale [or it is held with strong conviction, as defined by a score of 3 or more on the CASH rating scale for delusions]; or 4+ on conceptual disorganisation scale of BPRS)

- Duration of episode of less than 1 week

- Symptoms resolve spontaneously

- The brief limited psychotic symptom must have happened within the past year

Group 3: trait and state risk factors

- First-degree relative with a psychotic disorder or schizotypal personality disorder; or individual has schizotypal personality disorder

- Significant decrease in mental state or functioning: maintained for at least a month (reduction in GAF scale of 30 points from premorbid level)

- The decrease in functioning arose within the past year

\section{Exit criteria: acute psychosis}

- At least one of the following symptoms: hallucinations (defined by a score of 3 or more on the hallucinations scale of the BPRS); delusions (defined by a score of 4 or more on the unusual thought content scale of the BPRS or a score of 4 or more on the suspiciousness scale of the BPRS, or it is held with strong conviction, as defined by a score of 3 or more on the CASH rating scale for delusions or formal thought disorder [defined by a score of 4 or more on the conceptual disorganisation scale BPRS])

- Frequency of symptoms is at least several times a week

- Duration of mental state change is longer than 1 week

BPRS=brief psychiatric rating scale; $\mathrm{CASH}=$ comprehensive assessment of symptoms and history; GAF=global assessment of function scale. These are the criteria for identifying people as high risk for psychosis. People are included if they meet criteria for one or more of the three groups. Further details are provided elsewhere. ${ }^{9,10}$ Adapted from reference 9 with permission. 
ARTICLES

\begin{tabular}{|c|c|c|c|c|c|c|}
\hline & \multicolumn{3}{|l|}{ Cross-sectional analysis } & \multicolumn{3}{|l|}{ Longitudinal analysis } \\
\hline & $\begin{array}{l}\text { People who developed } \\
\text { psychosis }(n=23)\end{array}$ & $\begin{array}{l}\text { People who did not } \\
\text { develop psychosis }(n=52)\end{array}$ & $\mathrm{p}$ & $\begin{array}{l}\text { People who developed } \\
\text { psychosis }(n=10)\end{array}$ & $\begin{array}{l}\text { People who did not } \\
\text { develop psychosis }(n=11)\end{array}$ & $\mathrm{p}$ \\
\hline Sex (male/female) & $13 / 10$ & $30 / 22$ & 0.93 & $3 / 7$ & $4 / 7$ & $0 \cdot 76$ \\
\hline Age at baseline (years) & $19 \cdot 3(3 \cdot 7)$ & $21 \cdot 6(3 \cdot 3)$ & 0.009 & $18.9(4.5)$ & $20 \cdot 5(3 \cdot 7)$ & 0.29 \\
\hline Age at second scan (years) &.$\cdot$ &.$\cdot$ &.. & $20 \cdot 0(4.5)$ & $22 \cdot 3(4 \cdot 0)$ & $0 \cdot 14$ \\
\hline $\begin{array}{l}\text { NART-estimated premorbid IQ } \\
\text { at baseline* }\end{array}$ & $94.3(11 \cdot 9)$ & $95 \cdot 8(14.5)$ & $0 \cdot 70$ & $89 \cdot 9(12 \cdot 1)$ & $94 \cdot 0(14 \cdot 7)$ & 0.594 \\
\hline Time between scans (days) &.$\cdot$ &.$\cdot$ &.$\cdot$ & $374(180)$ & $637(382)$ & 0.04 \\
\hline $\begin{array}{l}\text { Days between baseline scan } \\
\text { and onset of psychosis }\end{array}$ & $200(215)$ &.$\cdot$ &.$\cdot$ & $172(213)$ & .. & .. \\
\hline $\begin{array}{l}\text { Days between onset of } \\
\text { psychosis and follow-up scan }\end{array}$ & $\cdot \cdot$ & .. & .. & $202(74)$ & .. &.$\cdot$ \\
\hline Duration of symptoms (days) $\dagger$ & $662(788)$ & 509 (884) & $0 \cdot 22$ & $610(570)$ & 318 (498) & $0 \cdot 27$ \\
\hline BPRS at intake & $20 \cdot 1(7 \cdot 8)$ & $19 \cdot 4(8 \cdot 4)$ & 0.73 & $20 \cdot 3(4 \cdot 9)$ & $19 \cdot 1(10 \cdot 5)$ & $0 \cdot 70$ \\
\hline SANS at intake & $29 \cdot 6(16 \cdot 3)$ & $24 \cdot 4(14 \cdot 9)$ & $0 \cdot 18$ & $27 \cdot 6(15 \cdot 8)$ & $22 \cdot 0(10 \cdot 0)$ & 0.57 \\
\hline
\end{tabular}

Data are mean (SD) unless otherwise stated. *Ten people (six with psychosis and four without) did not have NART data available at baseline. +Two people (one with psychosis and one without) did not have symptom-duration data available.

Table 1: Demographic data for the two groups of people

matter, white matter, cerebrospinal fluid, or dura/ vasculature) by a modified fuzzy clustering algorithm. ${ }^{11} \mathrm{We}$ applied this algorithm via a sliding window to the images, so that classification was adaptive to local variation in tissue contrast attributable to radio frequency or static field inhomogeneity, which can be a difficulty in the cerebellar region in particular. ${ }^{11}$ On the basis of previous findings, we assumed that the resulting probabilities of tissue-class membership could be equated with the proportional volumes of each tissue class in the often heterogeneous volume of tissue represented by each voxel. ${ }^{11}$ So, for example, if the probability of grey-matter-class membership was 0.8 for a particular voxel, then we assumed that $80 \%$ of the tissue represented by that voxel was grey matter.

To estimate between-group structural differences and within-group changes over time at each intracerebral voxel, we first co-registered the PD-weighted images from each fast spin-echo dataset with a template image in the standard space of Talairach and Tournoux by an affine transformation, ${ }^{12}$ implemented with the Fletcher-DavidonPowell algorithm. ${ }^{12}$ The template image was constructed by registering each of the images acquired from a subset of comparison participants in this study in standard space by an affine transformation, and then averaging these images. The affine transformation matrix that mapped each

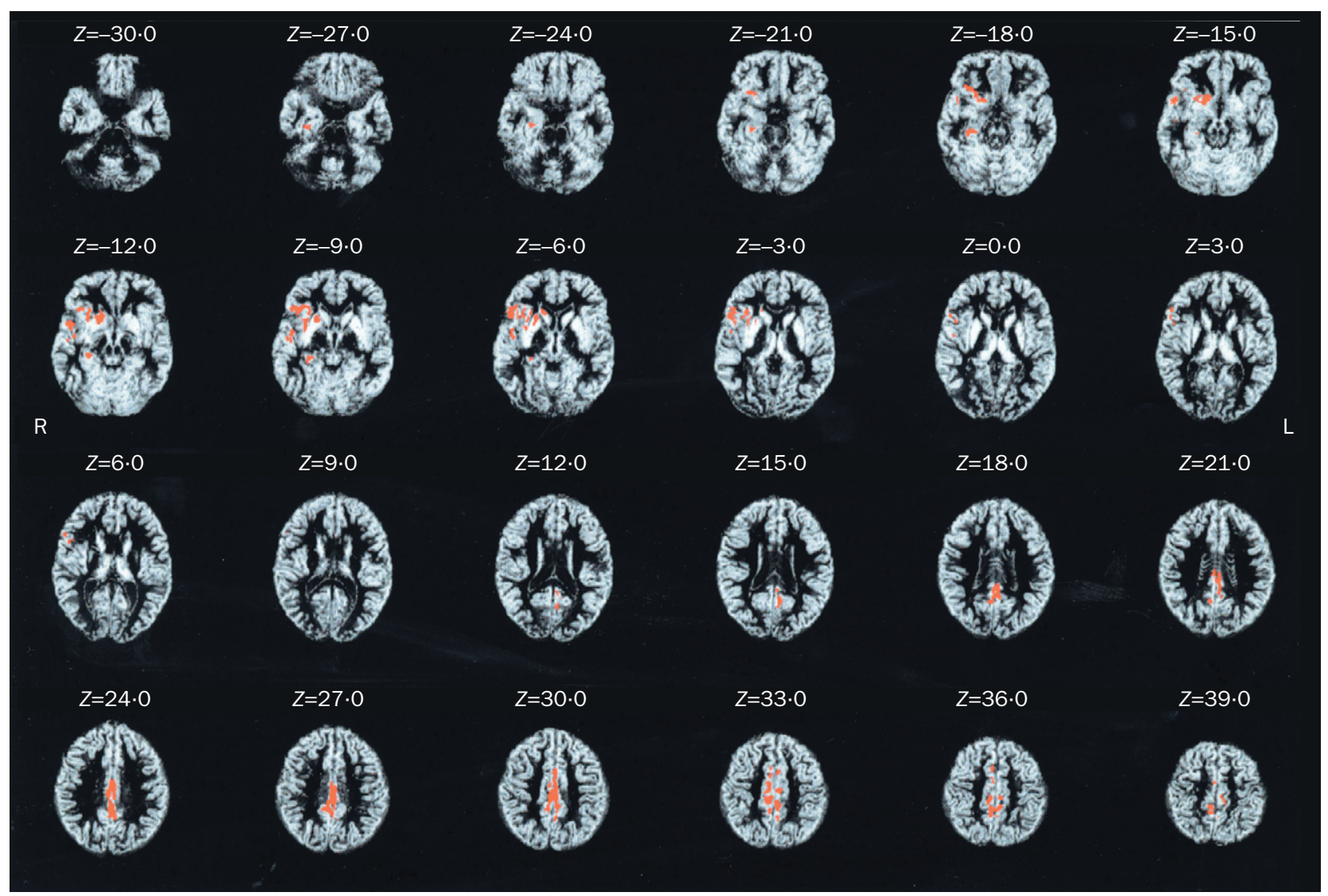

Figure 1: Grey-matter probability maps for baseline comparison of people who developed psychosis with those who did not

Red regions denote areas of reduced grey-matter volume in people who developed psychosis. Images are presented in standard radiological fashion, where right is left and vice versa. $Z$ coordinate shows position of each slice with respect to the Talairach atlas. Clusterwise probability of type I error, $p<0.004$, meaning less than one false-positive test is expected over the whole map. 


\begin{tabular}{|c|c|c|c|c|c|c|}
\hline & \multirow[t]{2}{*}{ Left or right } & \multirow[t]{2}{*}{ Brodmann's area } & \multicolumn{3}{|c|}{ Talairach coordinates of voxel of maximum significance } & \multirow[t]{2}{*}{ Size in voxels } \\
\hline & & & $x$ & $y$ & $z$ & \\
\hline Area & & & & & & \\
\hline $\begin{array}{l}\text { Hippocampus, parahippocampus, } \\
\text { and surrounding cortex }\end{array}$ & $\mathrm{R}$ & $28 / 35 / 36 / 38$ & 29 & -24 & -17 & 270 \\
\hline Superior temporal gyrus and temporal pole & $\mathrm{R}$ & $12 / 22 / 38$ & 49 & 2 & -11 & 300 \\
\hline $\begin{array}{l}\text { Inferior frontal gyrus, ventrolateral prefrontal } \\
\text { cortex, and basal ganglia }\end{array}$ & $\mathrm{R}$ & $44 / 45 / 47$ & 32 & 18 & -9 & 1428 \\
\hline Anterior and posterior cingulate & $L / R$ & $23 / 24 / 29 / 31 / 32$ & 2 & -29 & 28 & 2222 \\
\hline
\end{tabular}

individual's PD-weighted image onto this template was then applied identically to each of that person's four tissue-class probability maps to register them in standard space at the same voxel size as the original acquisition.

\section{Statistical analysis}

We estimated between-group differences in grey-matter volume by fitting an analysis of covariance (ANCOVA) model at each intracerebral voxel in standard space, which included age at scan and global grey-matter volume as covariates. We assessed within-group differences in grey matter with repeated-measures ANCOVA, also with global grey-matter volume and time between scans as covariates. Interaction terms (group by follow-up) were investigated to assess which areas showed significant differential changes between the groups over time (with time between scans and global grey-matter volume as covariates). In both cases, we modelled the effects of the covariates as simple linear effects on the grounds of parsimony.

We tested the null hypothesis by permutation at cluster level, as described in detail elsewhere. ${ }^{12}$ In brief, a map of the standardised ANCOVA model coefficient of interest $(\beta)$ at each voxel was thresholded such that if $\beta>2$ $(\operatorname{Prob}(\beta)<0 \cdot 05$, approximately) the voxel value was set to $\beta-2$, otherwise the voxel value was set to zero. This procedure generates a set of suprathreshold voxel clusters in three dimensions, each of which can be described in terms of its mass, or the sum of suprathreshold voxel statistics it comprises. The mass of each cluster was tested against a null distribution ascertained by repeated random permutation of the relevant factor in the ANCOVA model; refitting of the model and estimation of standardised coefficients at each voxel; and thresholding of the permuted coefficient maps to generate a set of 3-dimensional suprathreshold voxel clusters under the null hypothesis. The rationale for this non-parametric mode of inference is that test statistics for image analysis, which incorporate spatial information such as 3-dimensional cluster mass, are generally more powerful than other possible test statistics, such as $\beta$, which are informed only by data at a single voxel. Yet theoretical approximations to the null distribution of spatial statistics estimated in imaging data might be over-conservative or intractable. ${ }^{12}$ Here, we have consistently used probability thresholds for clusterwise testing such that the expected number of false-positive tests for each map is less than one.

Significant foci were anatomically localised with the standard atlas of Talairach and Tournoux, ${ }^{13}$ except for foci close to the cerebellum where we used the atlas of Schmahmann and colleagues, ${ }^{14}$ because the Talairach atlas does not show great anatomical detail in the cerebellar region.

Role of the funding source

The sponsor of the study had no role in study design, data collection, data analysis, data interpretation, or writing of the report.

\section{Results}

We enrolled 75 people for the cross-sectional comparison. All participants were aged 14-30 years and had never had an episode of frank psychosis. Of these, $23(31 \%)$ developed psychosis during follow-up, 18 within 12 months and a further five within 24 months. The remaining $52(69 \%)$ did not develop psychosis during the follow-up period. Follow-up was for at least 12 months: 67 (89\%) people were reassessed between 12 and 18 months; maximum follow-up was 44 months. The groups did not differ by sex, baseline BPRS, SANS, or NART score, duration of symptoms, or total corrected brain volume (table 1). However, participants who developed psychosis were significantly younger than those who did not develop this disorder $(p=0 \cdot 009)$.

In the 23 people who developed psychosis, the disorders were: schizophrenia $(n=8)$; schizoaffective disorder (2); brief psychotic episode (1); psychosis not otherwise specified (1); bipolar disorder with psychotic features (6); and major depression with mood incongruent psychotic disorder (4). One individual developed a psychosis with affective features (SCID diagnosis unavailable). 28 (54\%) of 52 individuals who did not develop a psychosis had no psychiatric diagnosis at follow-up. Of those who did meet criteria for a psychiatric disorder $(n=24), 11$ had a mood disorder (five with major depressive disorder; six with dysthymia); eight had an anxiety disorder (four social phobia; two general anxiety disorder; two panic disorder); three had obsessive-compulsive disorder; one had an eating disorder; and one had adjustment disorder.

$21(28 \%)$ of the 75 participants were included in the longitudinal comparison. These individuals were representative of the larger pool of ultra high-risk people we scanned at baseline in terms of all demographic and clinical variables (age, height, IQ, duration of symptoms, and BPRS and SANS ratings), apart from sex (more females than in the original group, $\mathrm{p}=0.012$ ), and they did not differ for whole brain volume. Ten $(48 \%)$ of the 21 participants became psychotic during follow-up, and 11 did not (table 1 ).

The diagnostic breakdown of psychotic disorders in patients rescanned in the longitudinal comparison (as defined with the SCID) was: schizophrenia $(n=5)$; schizoaffective disorder (1); brief psychotic episode (1); psychosis not otherwise specified (1); manic episode with psychotic features (1); and major depression with mood incongruent psychotic disorder (1). Six of 11 people who were not psychotic when rescanned in the longitudinal comparison had no psychiatric diagnosis at follow-up, whereas the remaining five were diagnosed with major depressive disorder, dysthymia, generalised anxiety disorder, eating disorder, and obsessivecompulsive disorder.

Most participants did not receive antipsychotic drugs, either before onset of psychosis (in those who developed 


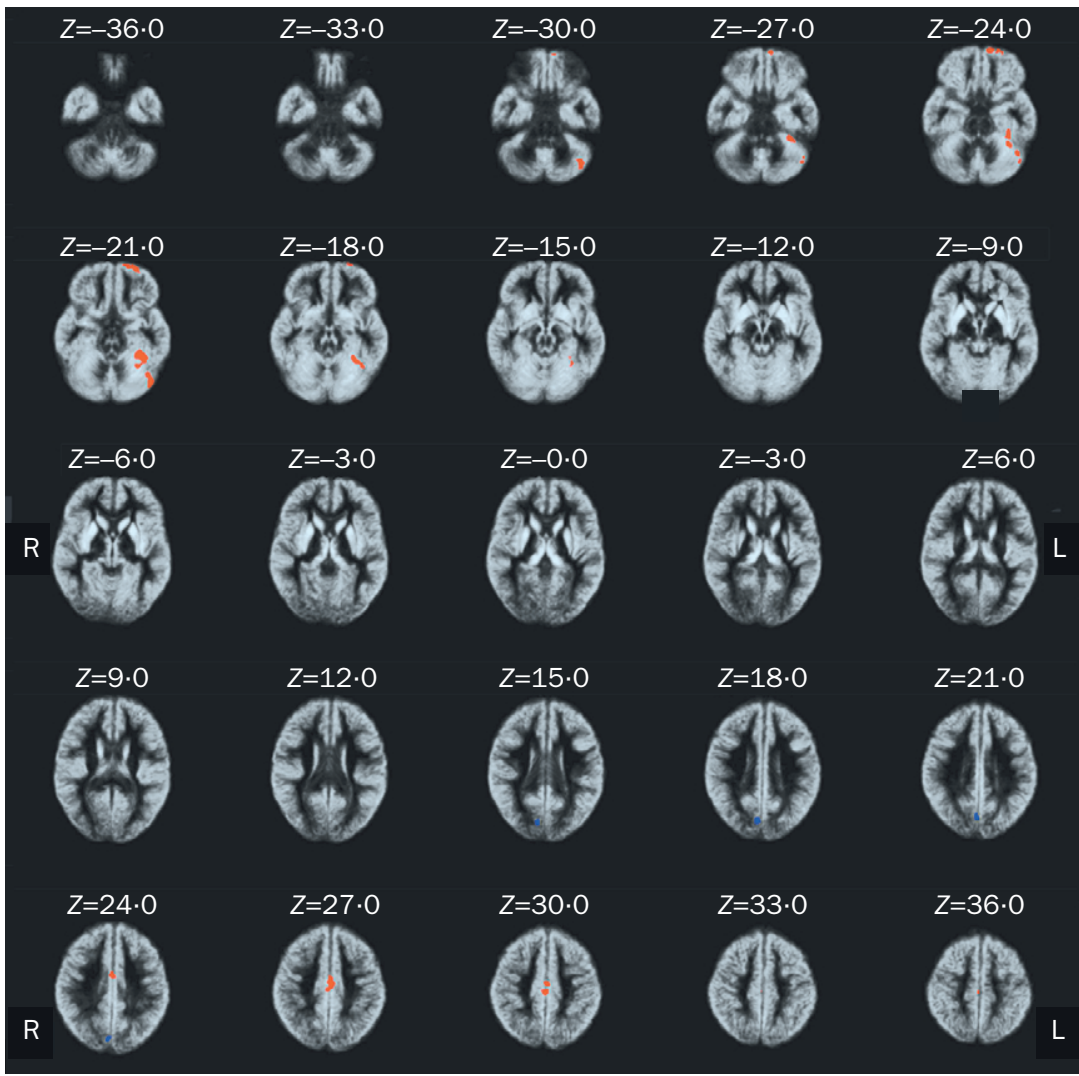

Figure 2: Grey-matter probability map for people who developed psychosis

Red regions denote areas of grey-matter reduction during follow-up, whereas blue regions denote areas of increase. Images are presented in standard radiological fashion, where right is left and vice versa.

$Z$ coordinate shows position of each slice with respect to the Talairach atlas. Clusterwise probability of type I error, $p<0.003$, meaning less than one false positive test is expected over the whole map.

psychosis) or by time of second MRI scan (in those without psychosis), except for three without psychosis and two with the disorder, who received 1-2 mg risperidone for up to 6 months after baseline scan. After onset of psychosis, all patients but one received atypical antipsychotic drugs (nine patients were treated with $1-3 \mathrm{mg}$ risperidone, one received trifluoperazine). One patient who was treated with risperidone did not adhere to treatment.

In the longitudinal comparison, participants who did or did not develop psychosis did not differ by age, sex, premorbid IQ, or baseline symptom ratings. However, the interval between scans was significantly longer for the ultra high-risk people who did not develop psychosis. The time between scans was therefore used as a covariate to remove any potential effects of differences in the interscan interval (table 1). A secondary analysis with age as the covariate did not substantially change these results.
In the cross-sectional comparison, participants who subsequently developed psychosis had smaller greymatter volumes at baseline in four regions than those who did not develop psychosis (figure 1 , table $2 ; \mathrm{p}<0 \cdot 004$ ). These regions comprised: a right medial temporal region, which included the hippocampus, and parahippocampal cortex; a right lateral temporal region, encompassing the superior temporal gyrus and temporal region; a right inferior frontal region, which included the orbital portion of the inferior frontal gyrus and the adjacent parts of the insula and basal ganglia; and a cingulate region, which included the anterior and posterior cingulate gyrus bilaterally. At a less conservative statistical threshold $(\mathrm{p}<0 \cdot 01)$, participants who subsequently developed psychosis also showed smaller grey-matter volumes in the left homologues of the first three regions, and in the dorsolateral prefrontal cortex.

In the longitudinal comparison, in people who developed psychosis, a significant bilateral reduction $(p<0.003)$ in grey-matter volume was noted between the baseline and followup MRI scans in the cingulate gyri. Further reductions in grey-matter volume were evident in the left parahippocampal gyrus, left fusiform gyrus, the left orbitofrontal cortex, and one region in the left cerebellar cortex (figure 2; table 3). One area of increased volume was recorded, in the right cuneus.

In participants who did not develop psychosis, the only significant difference between baseline and follow-up MRI scans was a reduction in volume in a similar region of the left cerebellum, as seen in those developing psychosis $(\mathrm{p}<0.003$; figure 3$)$.

Analysis of the interaction of group (psychotic vs nonpsychotic) by time (baseline scan vs follow-up scan) did not identify any significant regions ( $p$ set at $<0 \cdot 01$ ).

\section{Discussion}

Cross-sectional comparison of people at ultra high-risk for development of psychosis showed striking differences in regional grey-matter volume between those who subsequently developed a psychotic illness and those who did not, even though these subgroups were clinically

\begin{tabular}{|c|c|c|c|c|c|c|}
\hline & \multirow[t]{2}{*}{ Left or right } & \multirow[t]{2}{*}{ Brodmann's area } & \multicolumn{3}{|c|}{ Talairach coordinates of voxel of maximum significance } & \multirow[t]{2}{*}{ Size in voxels } \\
\hline & & & $x$ & $y$ & $z$ & \\
\hline \multicolumn{7}{|l|}{ People with psychosis } \\
\hline \multicolumn{7}{|l|}{ Decreases } \\
\hline Parahippocampal and fusiform gyri & L & $36 / 37 / 20$ & -31 & -42 & -25 & 416 \\
\hline Cerebellum & L & & -41 & -65 & -25 & 216 \\
\hline Cingulate gyrus & $\mathrm{L} / \mathrm{R}$ & $23 / 24 / 33$ & 1 & -14 & 28 & 221 \\
\hline Orbital frontal gyrus & L & 11 & -17 & 60 & -23 & 171 \\
\hline \multicolumn{7}{|l|}{ Increases } \\
\hline Cuneus & $\underline{\mathrm{R}}$ & 18 & 7 & -74 & 19 & 127 \\
\hline \multicolumn{7}{|l|}{ People without psychosis } \\
\hline \multicolumn{7}{|l|}{ Decreases } \\
\hline Cerebellum & $\mathrm{L}$ & & -31 & -74 & -26 & 233 \\
\hline
\end{tabular}

Table 3: Talairach coordinates and Brodmann's areas for changes in the groups of people between baseline and follow-up scans 


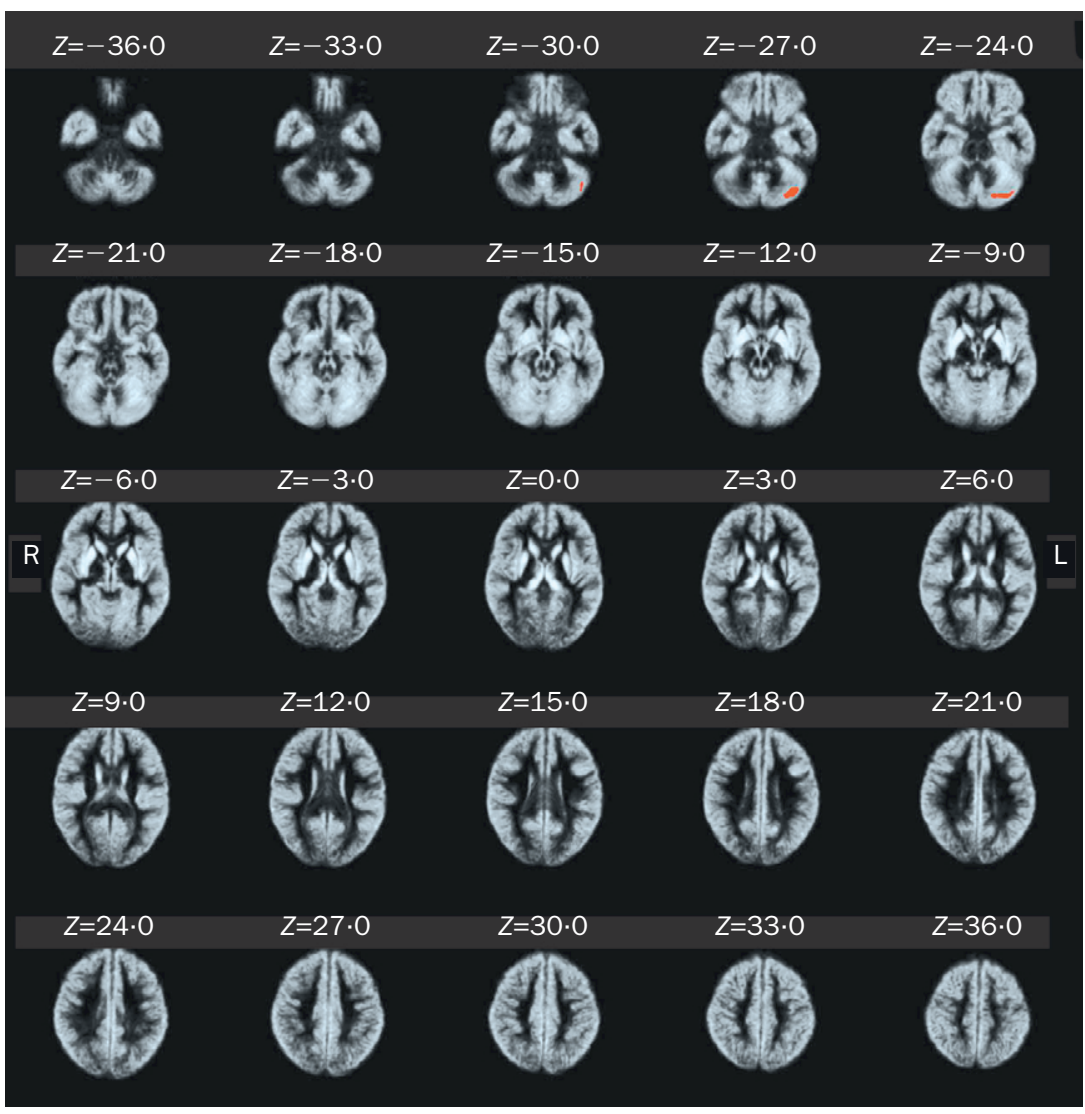

Figure 3: Grey-matter probability map for people who did not develop psychosis Red regions denote areas of grey-matter reduction during follow-up. Images are presented in standard radiological fashion, where right is left and vice versa. $Z$ coordinate shows position of each slice with respect to the Talairach atlas. Clusterwise probability of type I error, $p<0.003$, meaning less than one false positive test is expected over the whole map.

indistinguishable at the time of scanning. Thus, in this high-risk sample, smaller volumes of grey matter in the right prefrontal cortex, insular, and temporal cortex, the right basal ganglia, and the cingulate cortex bilaterally were associated with subsequent development of psychosis.

Second, follow-up MRI scanning in ultra high-risk individuals indicated that further structural brain changes arose in inferior frontal, cingulate, and temporal lobe regions between the prodromal phase and first expression of frank psychosis. These longitudinal changes were not evident in ultra high-risk participants who did not subsequently develop psychosis.

Our findings suggest that people who will subsequently develop psychotic disorders have neuroanatomical abnormalities (especially in the right hemisphere) that predate the first episode of frank psychosis. However, our data also suggest that there could be further changes in grey-matter volume that appear in association with first expression of frank psychosis, although whether these changes represent the cause or the effects of psychosis is unclear.

We intended to scan all ultra high-risk people developing psychosis as soon as possible after onset of their disorder, whereas those who did not develop psychosis were scanned after 12-month follow-up had elapsed. However, in practice we could not always contact these people at the desired timepoint, resulting in a large range in the interscan interval. This was controlled for in the analysis, suggesting that the changes identified related to making the transition to frank psychosis.
Abnormalities evident before onset of psychosis cannot be ascribed to effects of antipsychotic drugs, because none of the participants was receiving drugs at that time. It is possible that the longitudinal changes evident in patients with psychosis were related to treatment with antipsychotic drugs after onset of the disorder. However, although typical antipsychotics can increase striatal volume, ${ }^{15}$ there is little evidence at present that atypical drugs can cause reductions in grey-matter volume in the cerebral cortex. Nevertheless, further work in patients who have not taken drugs will be necessary to resolve this issue.

The cross-sectional and longitudinal MRI differences are unlikely to reflect alcohol use, since this use was minimal in both groups. Similarly, although three patients in the psychotic high-risk group used significant quantities of cannabis (compared with none in the nonpsychotic group), there is no evidence that cannabis use is associated with MRI grey-matter changes. ${ }^{16}$

In the cross-sectional comparison, individuals who subsequently became psychotic were slightly younger than those who did not, but age was used as a covariate in the analysis. In the longitudinal comparison, the ages between groups were similar, and a secondary analysis adjusting for age had no effect on our results. Moreover, prospective MRI studies in normal adolescents have not identified significant volumetric changes in medial temporal lobe structures. ${ }^{17}$ It is important to note that our results were in ultra high-risk individuals with prodromal symptoms, who might not be representative of all people who develop a psychotic illness.

We used a voxel-based automated image analysis method that keeps operator bias to a minimum, and permits analysis of the whole brain, rather than a few regions of interest. Although there has been some debate about the relative merits of this approach compared with region of interest techniques, there is good correlation between results from these methods, ${ }^{18}$ and voxel-based analyses have produced relatively consistent results in studies of patients with established schizophrenia, ${ }^{18,19}$ though few such studies have been reported in other psychoses.

The number of participants in our study precluded separate analyses by disorder, but most of those who developed psychosis met diagnostic criteria for schizophrenia spectrum disorder. However, these diagnoses should be deemed provisional, because they were made at a relatively early stage of the disorder and might need revision in view of subsequent episodes.

Patients with established schizophrenia and bipolar disorder show qualitatively similar MRI abnormalities, which include volume reductions in regions identified in our study, ${ }^{3-6}$ though there might also be differences-eg, larger amygdalae in patients with bipolar disorder. ${ }^{20}$ These similarities, together with their overlapping psychopathologies - as well as evidence that psychopathological dimensions are better predictors of response to treatment ${ }^{21}$ and clinical course ${ }^{22}$ than diagnostic categories - and data 
suggesting cosegregation of familial risk for schizophrenia and bipolar disorder ${ }^{23}$ have prompted some authorities to conclude that these disorders form part of a continuum of psychosis. $^{24}$ Our results could thus inform our understanding of psychosis in general and be relevant to both schizophrenia and bipolar disorder. Further work will be necessary to investigate the extent to which the findings are associated with specific psychotic disorders.

At baseline, reduced grey-matter volumes were noted in individuals who later went on to develop psychosis in the prefrontal, cingulate, and to a lesser extent, temporal cortex. These regions have consistently been implicated in neuroimaging, ${ }^{3-6,19,25}$ neuropsychological, ${ }^{26}$ and neuropathological studies ${ }^{27}$ of patients with psychosis. Neuroimaging abnormalities in these regions have also been described in people at increased risk of psychosis for other reasons, such as the probands of monozygotic twin pairs discordant for schizophrenia, ${ }^{28}$ first-degree relatives of patients with psychosis, ${ }^{7}$ and individuals with schizotypal personality disorder. ${ }^{29}$

The main areas of the brain that showed longitudinal changes in patients who developed psychosis were the left medial temporal and the left orbitofrontal cortices, and bilaterally in the cingulate cortex, with the most extensive changes in the left medial temporal region. All these regions have been implicated in psychotic disorders, and metaanalyses of neuroimaging studies suggest that the medial temporal region is the site of the most robust abnormalities in schizophrenia. ${ }^{25}$ Although there is also evidence that the cerebellum is implicated in schizophrenia, ${ }^{25,30}$ the longitudinal differences in the left cerebellum were noted in people with and without psychosis, suggesting that this change was not related to onset of the disorder. The findings in the cuneus were not predicted and are difficult to interpret, although this region has been shown to be hypoactive in functional imaging studies of schizophrenia. ${ }^{30}$

Our data suggest that progressive changes in psychosis could arise at different sites to areas in which nonprogressive abnormalities are seen. This finding accords with data from Cannon and colleagues, ${ }^{28}$ who reported that the genetic vulnerability to schizophrenia in twins was associated with reduced prefrontal cortical thickness, whereas the psychotic phenotype was associated with reduced thickness in temporal and parietal cortex. An association between temporal abnormalities and expression of psychosis would also accord with data from crosssectional comparisons of first-episode psychotic and highrisk groups. ${ }^{7,9}$ These observations, and our data, suggest that volumetric changes might develop in certain cortical regions, such as the medial temporal lobe, during the transition to psychosis. Whether these changes represent loss of grey matter or changes in the biochemical composition of the tissue cannot be established from our study, and would need to be investigated with other techniques.

It is important to note that although the cross-sectional MRI differences we noted between ultra high-risk individuals who did and did not subsequently develop psychosis suggest that some neuroanatomical abnormalities predate expression of psychosis, they could also have resulted from an earlier progressive process. However, this possibility seems unlikely, because the duration of prodromal symptoms at the time of the baseline scan did not differ between the groups who did and did not go on to develop psychosis. This issue could be clarified further by serial examination of individuals within the prodromal phase.

Our cross-sectional data raise the possibility that MRI or other investigations could help to distinguish ultra high- risk people who will subsequently develop psychosis from those who will not, although at present it is still too early to say whether MRI will prove to be usable as a diagnostic tool. The fact that progressive changes might be happening before onset of frank psychotic symptoms has important implications for treatment of psychotic disorders. At present, treatment is normally withheld until the clinician is confident that the patient has a psychotic disorder, although there is evidence that the longer the delay before initiating treatment, the poorer the prognosis. ${ }^{31}$ Our data suggest that neuroanatomical changes might be happening before or during the transition to psychosis, raising the possibility that intervention might be indicated before expression of frank psychotic symptoms, ${ }^{32}$ and might have an effect on the underlying pathological process.

\section{Contributors}

C Pantelis, D Velakoulis, S J Wood, and P D McGorry had a role in study design, supervision of staff involved in data collection at all stages, data analysis, and drafting of the report. L J Phillips, A R Yung, and W Brewer assessed all participants clinically, coordinated MRI scanning, and participated at all stages of development of the final report. $\mathrm{P}$ Desmond was the radiologist involved in MRI scanning, reported on all scans, and assisted with the report. B Soulsby undertook the automated analysis in close collaboration with J Suckling and $\mathrm{E}$ T Bullmore, and commented on various drafts of the report. J Suckling, E T Bullmore, and P K McGuire were involved in design, analysis, and drafting of the final report. All co-authors have seen and approved the final version of the report.

\section{Conflict of interest statement}

None declared.

\section{Acknowledgments}

This research, and the clinical research structure of the personal assessment and crisis evaluation (PACE) clinic in Melbourne, Australia, was supported by project grants from the National Health and Medical Research Council (NHMRC; grant ID numbers 970598 and 970391); Victorian Health Promotion Foundation; the Stanley Foundation, Ian Potter Foundation; and Woods Family Trust. D Velakoulis and S J Wood were supported as Research Officers with funding from the NHMRC. P D McGorry was supported by a National Alliance for Research on Schizophrenia and Depression distinguished investigator award, and E T Bullmore was supported by the Wellcome Trust.

\section{References}

1 McGorry PD, Copolov DL, Singh BS. Current concepts in functional psychosis: the case for a loosening of associations. Schizophr Res 1990; 3: 221-34.

2 Weinberger DR, McClure RK. Neurotoxicity, neuroplasticity, and magnetic resonance imaging morphometry: what is happening in the schizophrenic brain? Arch Gen Psychiatry 2002; 59: 553-58.

3 Elkis H, Friedman L, Wise A, Meltzer HY. Meta-analyses of studies of ventricular enlargement and cortical sulcal prominence in mood disorders: comparisons with controls or patients with schizophrenia. Arch Gen Psychiatry 1995; 52: 735-46.

4 Videbech P. MRI findings in patients with affective disorder: a metaanalysis. Acta Psychiatr Scand 1997; 96: 157-68.

5 Roy PD, Zipursky RB, Saint-Cyr JA, Bury A, Langevin R, Seeman MV. Temporal horn enlargement is present in schizophrenia and bipolar disorder. Biol Psychiatry 1998; 44: 418-22.

6 Velakoulis D, Pantelis C, McGorry PD, et al. Hippocampal volume in first-episode psychoses and chronic schizophrenia: a highresolution magnetic resonance imaging study. Arch Gen Psychiatry 1999; 56: 133-40.

7 Lawrie SM, Whalley H, Kestelman JN, et al. Magnetic resonance imaging of brain in people at high risk of developing schizophrenia. Lancet 1999; 353: 30-33.

8 Wood SJ, Velakoulis D, Smith D, et al. A longitudinal study of hippocampal volume in first episode psychosis and chronic schizophrenia. Schizophr Res 2001; 52: 37-46.

9 Phillips LJ, Velakoulis D, Pantelis C, et al. Non-reduction in hippocampal volume is associated with higher risk of psychosis. Schizophr Res 2002; 58: 145-58.

10 Yung AR, Phillips LJ, McGorry PD, et al. Prediction of psychosis: a step towards indicated prevention of schizophrenia. Br F Psychiatry 1998; 172 (suppl 33): 14-20.

11 Suckling J, Sigmundsson T, Greenwood K, Bullmore ET. A modified fuzzy clustering algorithm for operator independent tissue 
classification of dual echo MR images. Magn Reson Imaging 1999; 17: 1065-76.

12 Bullmore ET, Suckling J, Overmeyer S, Rabe-Hesketh S, Taylor E, Brammer M. Global, voxel and cluster tests, by theory and permutation for a difference between two groups of structural MR images of the brain. IEEE Trans Med Imaging 1999; 18: 32-42.

13 Talairach J, Tournoux P. Co-planar stereotaxic atlas of the human brain. New York: Thieme Medical Publishers, 1988.

14 Schmahmann JD, Doyon J, McDonald D, et al. Three-dimensional MRI atlas of the human cerebellum in proportional stereotaxic space. Neuroimage 1999; 10: 233-60.

15 Keshavan MS, Bagwell WW, Haas GL, Sweeney JA, Schooler NR, Pettegrew JW. Changes in caudate volume with neuroleptic treatment. Lancet 1994; 344: 1434.

16 Aasly J, Storsaeter O, Nilsen G, Smevik O, Rinck P. Minor structural brain changes in young drug abusers: a magnetic resonance study. Acta Neurol Scand 1993; 87: 210-14.

17 Rapoport JL, Giedd JN, Blumenthal J, et al. Progressive cortical change during adolescence in childhood-onset schizophrenia: a longitudinal magnetic resonance imaging study. Arch Gen Psychiatry 1999; 56: 649-54.

18 Wright IC, Ellison Z, Sharma T, Friston KJ, Murray RM, McGuire PK. Mapping of grey matter changes in schizophrenia. Schizophr Res 1999; 35: 1-14.

19 Sigmundsson T, Suckling J, Maier M, et al. Structural abnormalities in frontal, temporal, and limbic regions and interconnecting white matter tracts in schizophrenic patients with prominent negative symptoms. Am f Psychiatry 2001; 158: 234-43.

20 Altshuler LL, Bartzokis G, Grieder T, et al. An MRI study of temporal lobe structures in men with bipolar disorder or schizophrenia. Biol Psychiatry 2000; 48: 147-62.

21 Johnstone EC, Frith CD, Crow TJ, et al. The Northwick Park 'functional' psychosis study: diagnosis and outcome. Psychol Med 1992; 22: 331-46.
22 van Os J, Jones P, Sham P, Bebbington P, Murray RM. Psychosis as a continuum of variation in dimensions of psychopathology. In: Haffner H, Gattaz W, eds. Search for the causes of schizophrenia. Berlin: Springer, 1999: 59-80

23 Cardno AG, Rijsdijk FV, Sham PC, Murray RM, McGuffin P. A twin study of genetic relationships between psychotic symptoms. Am $f$ Psychiatry 2002; 159: 539-45.

24 Crow TJ. Nature of the genetic contribution to psychotic illness: a continuum viewpoint. Acta Psychiatr Scand 1990; 81: 401-08.

25 Wright IC, Rabe-Hesketh S, Woodruff PW, David AS, Murray RM, Bullmore ET. Meta-analysis of regional brain volumes in schizophrenia. Am f Psychiatry 2000; 157: 16-25.

26 Pantelis C, Wood SJ, Maruff P. Schizophrenia. In: Owen AM, Harrison J, eds. Cognitive deficits in brain disorders. London: Martin Dunitz, 2001: 217-48.

27 Harrison PJ. The neuropathology of schizophrenia: a critica review of the data and their interpretation. Brain 1999; 122: 593-624.

28 Cannon T, Thompson P, van Erp T, et al. A probabilistic atlas of cortical gray matter changes in monozygotic twins discordant for schizophrenia. Neuroimage 2001; 13: S1034.

29 Dickey CC, McCarley RW, Voglmaier MM, et al. Schizotypal personality disorder and MRI abnormalities of temporal lobe gray matter. Biol Psychiatry 1999; 45: 1393-402.

30 Crespo-Facorro B, Paradiso S, Andreasen NC, et al. Recalling word lists reveals "cognitive dysmetria" in schizophrenia: a positron emission tomography study. Am f Psychiatry 1999; 156: 386-92.

31 Wyatt R. Early intervention for schizophrenia: can the course of the illness be altered? Biol Psychiatry 1995; 38: 1-3.

32 McGorry PD, Yung AR, Phillips LJ, et al. Randomized controlled trial of interventions designed to reduce the risk of progression to first-episode psychosis in a clinical sample with sub threshold symptoms. Arch Gen Psychiatry 2002; 59: 921-28. 